\title{
Pre-Descemetic Placement of Intracorneal Ring Segments for Keratoconus after Mechanical Implantation
}

\author{
Faady Yahya ${ }^{a}$ Christian F. Prünte ${ }^{a, b}$ Hendrik P.N. Scholla, b \\ Zisis Gatzioufas a, b \\ aDepartment of Ophthalmology, University of Basel, Basel, Switzerland; 'bnstitute of \\ Molecular and Clinical Ophthalmology Basel (IOB), Basel, Switzerland
}

\section{Keywords}

Intracorneal ring segments · Keratoconus - Refractive surgery

\section{Abstract}

We report the case of a 23-year-old male who was referred to our clinic for a routine followup examination. The patient was treated for keratoconus 3 years ago in Colombia, where intracorneal ring segments were implanted mechanically in both eyes to improve his visual acuity. Surprisingly, we discovered a pre-descemetic placement of intracorneal ring segments, which could lead to potential complications, under circumstances. We illustrate this impressive finding with slit-lamp photography and high-resolution anterior segment OCT and advocate for the use of femtosecond laser as the method of choice for intracorneal ring segment insertion in order to provide maximal precision and safety.

\section{Introduction}

Intracorneal ring segments (ICRS) are synthetic spacer elements which are implanted within the cornea to improve its refractive properties in corneal ectatic eye disease such as keratoconus. The arcuate segments flatten the anterior corneal curvature by shortening the 

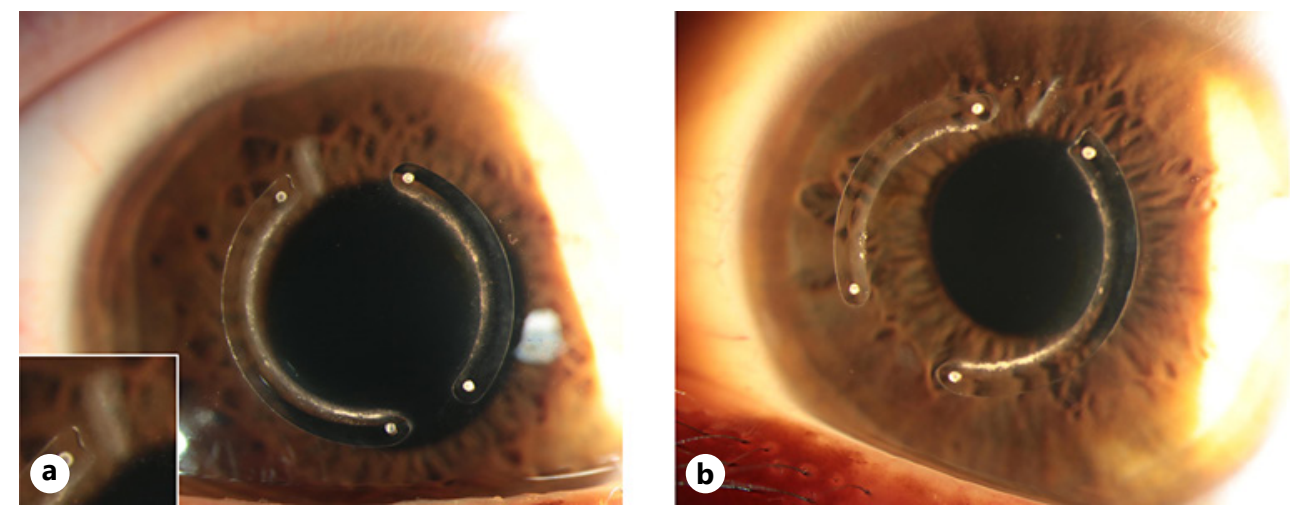

Fig. 1. Slit-lamp photograph of the (a) right and (b) left eye 3 years after ICRS implantation showing 2 ring segments in each eye and clustered deposits with a crystalline appearance particularly at the inner curvature of the ICRS. Inlay: Magnification of the proximal breakage site of the ring segment in the right cornea.

arc length of the optical zone when inserted at $70-80 \%$ of stromal depth, thereby improving visual acuity $[1,2]$.

The minimally invasive surgical technique consists of 3 steps: intrastromal channel formation, ring segment insertion, and entry site closure. In recent years, an essential advancement was made regarding surgical procedure safety and reduction of intra- and postoperative complications by performing femtosecond laser-assisted intracorneal incision and channel formation instead of manual stromal dissection $[3,4]$.

\section{Case Presentation}

We present a case of a 23-year-old Colombian male who was referred to our department for a routine follow-up examination after bilateral ICRS (Keraring ${ }^{\circledR}$; Mediphacos, Belo Horizonte, Brazil) implantation for keratoconus in Colombia in 2018. The patient did not undergo corneal crosslinking.

Slit-lamp photography and high-resolution anterior segment OCT (CASIA $2^{\circledR}$; Tomey Corporation, Nagoya, Japan) images revealed that ICRS were implanted into the predescemetic corneal space possibly after improper mechanical stromal dissection (shown in Fig. 1, 2). We also observed clustered deposits with a crystalline appearance and fibrotic response in the stromal channels, particularly along the inner curvature of the ICRS (shown in Fig. 1). Furthermore, a breakage of their proximal endings was visible (shown in Fig. 1 Inlay). Anterior segment OCT confirmed the deep placement of all ICRS into the pre-descemetic layer of the cornea (shown in Fig. 2). Notably, the angle position of ICRS inserted in the temporal corneal side was misaligned and formed a prominent endothelial step leading to retro-corneal protrusion (shown in Fig. 1, 2). Endothelial specular microscopy was performed to determine endothelial cell morphology and density, demonstrating normal findings: The endothelial cell density of the right eye was measured $2,234 / \mathrm{mm}^{2}$ with a standard deviation of $181{\mu \mathrm{m}^{2}}^{2}$ of the left eye $2,569 / \mathrm{mm}^{2}$ with a standard deviation of $140 \mu \mathrm{m}^{2}$.

The best corrected visual acuity (BCVA) was found to be equal on both eyes at 0.63 after autorefraction correction for the right eye $\left(-3.25 \mathrm{sph} /-1.00 \mathrm{cyl} / 3^{\circ} \mathrm{ax}\right)$ and left eye $(-1.75$ $\mathrm{sph} /-2.75 \mathrm{cyl} / 176^{\circ} \mathrm{ax}$ ). Keratography measurement performed by anterior segment OCT revealed for the right and left eye a Kmax of 45.4D and 53.3D, respectively.

\section{Karger's}




\section{Case Reports in Ophthalmology}

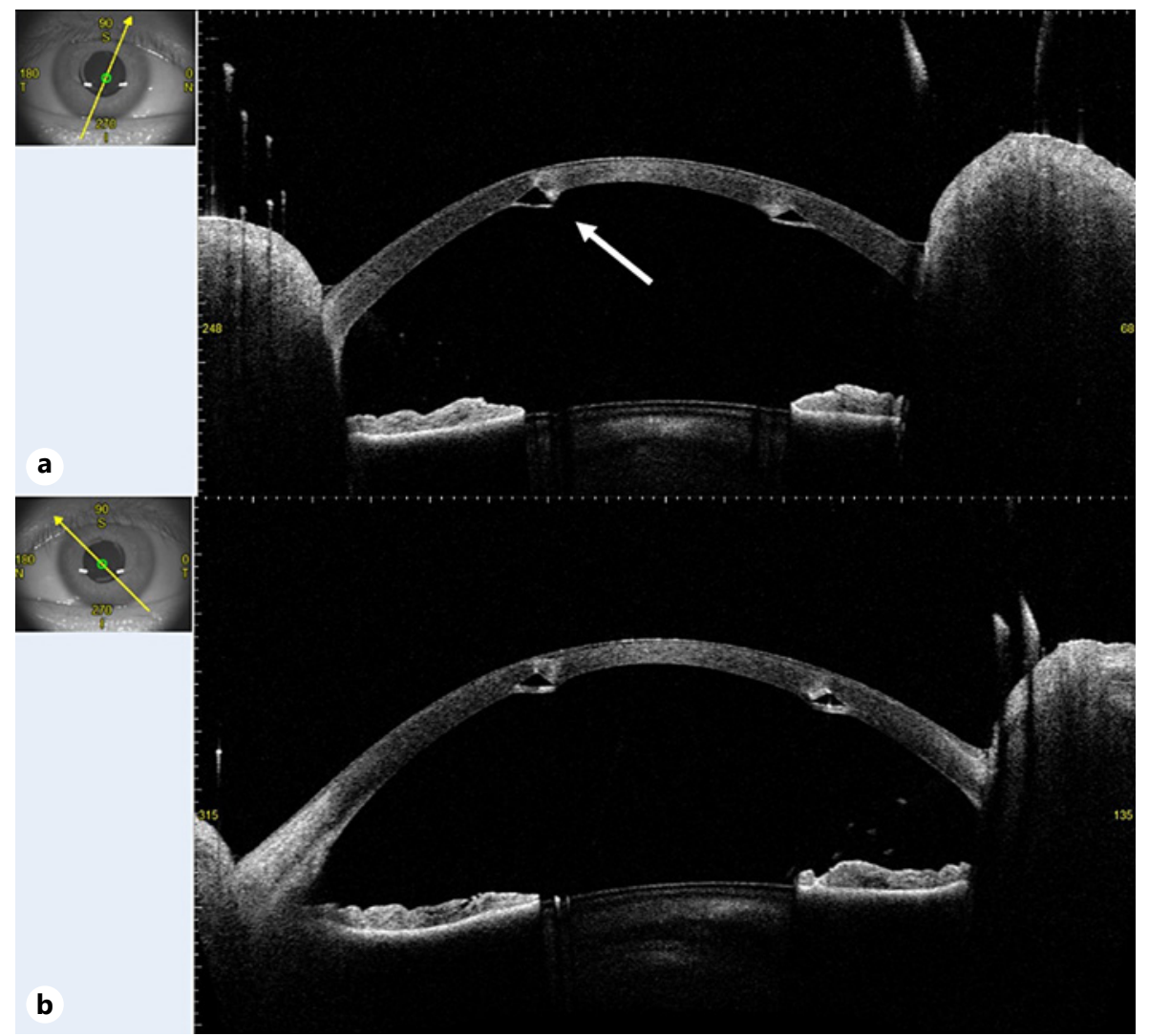

Fig. 2. Corresponding anterior segment OCT (CASIA $2^{\circledR}$ ) images for the (a) right and (b) left eye confirm a pre-descemetic position of all 4 triangular ICRS and angle misalignment leading to a prominent endothelial step formation and consequent retro-corneal protrusion, exemplarily shown on the right temporal side (white arrow).

\section{Discussion/Conclusion}

In our case, mechanical ICRS implantation was associated with suboptimal intracorneal placement of the ring segments and angle misalignment possibly due to improper plane dissection. Excessive eye rubbing, blunt trauma exposure, or simply keratoconus progression could lead to descemetic ICRS extrusion followed by endothelial rupture and segment migration into the anterior chamber.

Consequently, in our opinion, the femtosecond laser-assisted technique for ICRS insertion warrants maximal precision and reproducibility and should represent the method of choice in ICRS implantation for keratoconus. Preferably, it should be combined with corneal crosslinking to stabilize the refractive outcome provided by ICRS implantation [5].

\section{Statement of Ethics}

In accordance with the guidelines of our institute, single case reports are exempt from requiring ethics approval. The study has been performed in compliance with the Helsinki Declaration. The authors certify that they have obtained all appropriate patient consent forms. In the form, the patient has given his informed consent for his images and other clinical information to be reported in the journal. The patient understands that his name and initial 
will not be published and due efforts will be made to conceal his identity, but anonymity cannot be guaranteed.

\section{Conflict of Interest Statement}

The authors have no conflicts of interest to declare.

\section{Funding Sources}

This manuscript did not receive any funding.

\section{Author Contributions}

F.Y. contributed to the report conception and data acquisition and analysis and wrote the manuscript. F.Y., H.P.N.S., C.F.P., and Z.G. contributed to data interpretation and manuscript revision. All authors have read and approved the final manuscript and agreed to be accountable for the author's own contributions and ensure the accuracy or integrity of any part of the work.

\section{Data Availability Statement}

Data and material are available upon reasonable request.

\section{References}

1 Colin J, Cochener B, Savary G, Malet F. Correcting keratoconus with intracorneal rings. J Cataract Refract Surg. 2000;26(8):1117-22.

2 Sakellaris D, Balidis M, Gorou O, Szentmary N, Alexoudis A, Grieshaber MC, et al. Intracorneal ring segment implantation in the management of keratoconus: an evidence-based approach. Ophthalmol Ther. 2019;8: $5-14$.

3 Tiago M, Alfonso JF, Freitas R, Franqueira N, Faria-Correira F, Ambrosio R, et al. Comparison of complication rates between manual and femtosecond laser-assisted techniques for intrastromal corneal ring segments implantation in keratoconus. Curr Eye Res. 2019;44(12):1291-8.

4 Kubaloglu A, Sari ES, Cinar Y, Cingu K, Koytak A, Coşkun E, et al. Comparison of mechanical and femtosecond laser tunnel creation for intrastromal corneal ring segment implantation in keratoconus: prospective randomized clinical trial. J Cataract Refract Surg. 2010;36(9):1556-61.

5 Hashemi H, Alvani A, Seyedian MA, Yaseri M, Khabazkhoob M, Esfandiari H. Appropriate sequence of combined intracorneal ring implantation and corneal collagen cross-linking in keratoconus: a systematic review and meta-analysis. Cornea. 2018;37(12):1601-7. 